\title{
2||-||||||||||||||||||||||||||||||||||||||||||||||||||||||||||||||||.
}

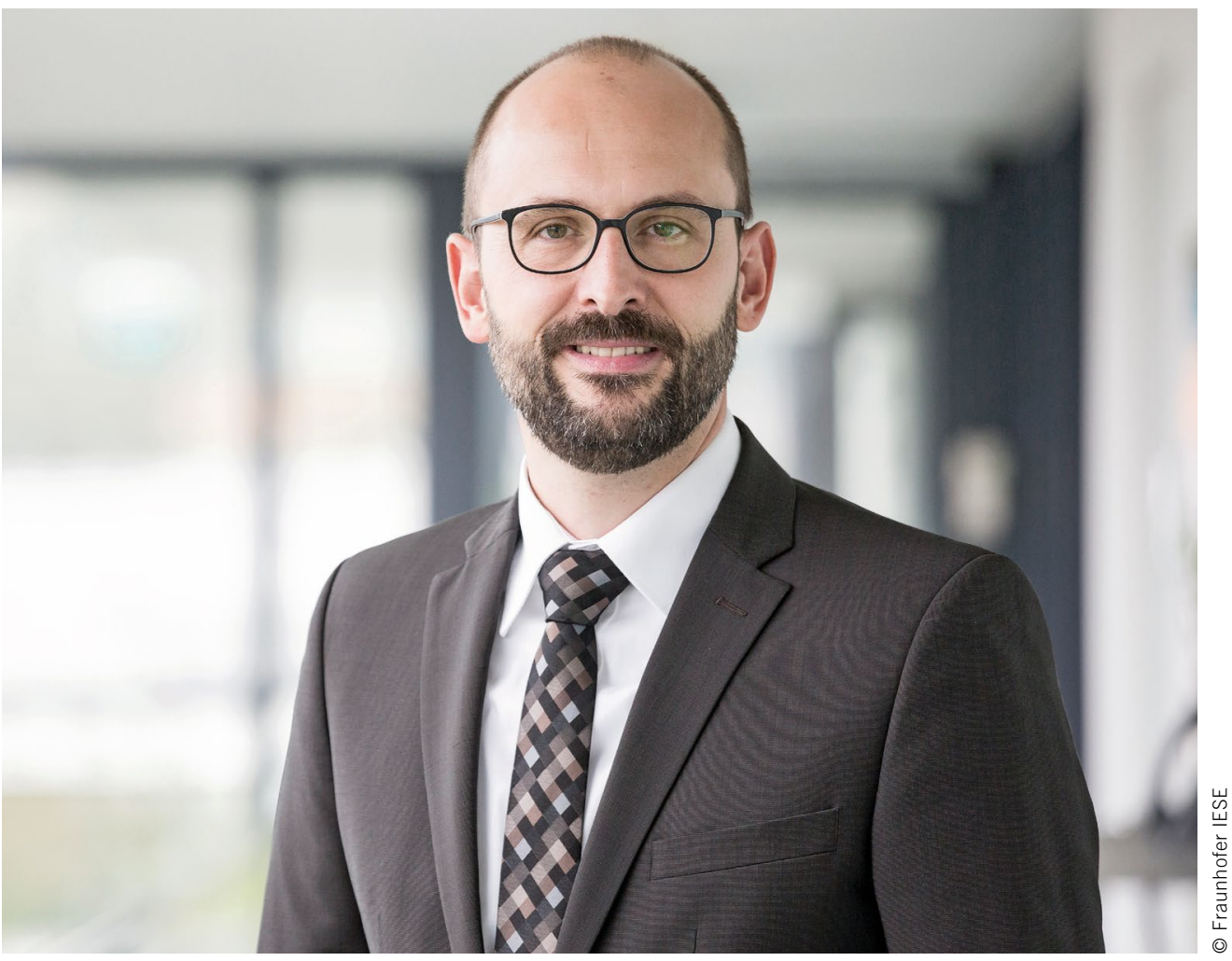

Prof. Dr.-Ing. Jörg Dörr

Head of the Digital Farming Chair at Technische Universität Kaiserslautern, and Member of the Extended Institute Management of Fraunhofer IESE in Kaiserslautern (Germany)

\section{Digital Farming in the Context of Digital Ecosystems}

Biological ecosystems in agriculture are complex. Plants, soil, animals, climate, etc. interact in many different ways. However, the complexity of the digital agricultural ecosystem should not be underestimated in the meantime! To address the challenges farmers face, there are now thousands of digital farming solutions, cyber-physical systems with (partially) autonomous functions in, on, and behind the tractor, intelligent combine harvesters, drones, various farm management information systems, and mobile apps, to name just a few. All these systems should not be considered alone, but in their role in the agricultural production process on the field and in the digital agricultural ecosystem: what contribution do these solutions make on their own, but especially in their interaction with all other solutions in the domain?

The interoperability of systems, that is to say, their ability to easily interact with each other, through their primary functionality or through the usage of each other's data, will be a key to the success of digital farming. Too little attention is being paid to this, even though farmers have long complained about the lack of interoperability and concerns about vendor lock-in.
Have all the concepts for truly interoperable systems really been sufficiently developed - for example, in terms of control over data? Current initiatives in the agricultural domain, such as research projects in the area of GAIA-X or concepts for an Agricultural Data Space with digital twins and data usage control, promise to make a valuable contribution here.

Let's get back to the benefits of the systems: what is the use of a nitrogen sensor? Why is it worth the purchase? Digital farming solutions bring a significant contribution to solving the challenges of our society and farms for stable yield, for sustainability, and for food quality. Digital farming will bring benefits to small and large farms alike. Every farmer should take a close look at the new possibilities. Research and development also have a lot to do to make the contributions of digital farming solutions clearer and not to see them just one-dimensionally. Otherwise, who would be surprised if market acceptance falls short of expectations? It's hard for farmers to gain or maintain an overview. There is a lot to do. Because an ecosystem thrives on interaction. This also applies to (digital) agriculture. 